\title{
Campaniforme (céramique)
}

G. Souville

\section{(2) OpenEdition}

Journals

Édition électronique

URL : http://journals.openedition.org/encyclopedieberbere/2086

DOI : 10.4000/encyclopedieberbere.2086

ISSN : 2262-7197

\section{Éditeur}

Peeters Publishers

\section{Édition imprimée}

Date de publication : 1 septembre 1992

Pagination : 1725-1728

ISBN : 2-85744-581-4

ISSN : 1015-7344

\section{Référence électronique}

G. Souville, «Campaniforme (céramique) », Encyclopédie berbère [En ligne], 11 | 1992, document C13, mis en ligne le 01 avril 2013, consulté le 24 septembre 2020. URL : http://journals.openedition.org/ encyclopedieberbere/2086; DOI : https://doi.org/10.4000/encyclopedieberbere.2086

Ce document a été généré automatiquement le 24 septembre 2020

(c) Tous droits réservés 


\title{
Campaniforme (céramique)
}

\author{
G. Souville
}

1 La céramique campaniforme caractérisée par des gobelets en forme de cloche, par un décor à bandes horizontales incisées ou impressionnées au peigne est très répandue en Europe occidentale et en Europe centrale. Elle est généralement associée à des poignards en cuivre, des boutons en os et des brassards d'archer en pierre. Très abondante dans la péninsule Ibérique où l'on avait cru voir l'origine de cette culture, elle est fréquemment recueillie, dans des tombes individuelles.

2 Le Campaniforme est largement représenté au Maroc. Pressenti par Armand Ruhlmann, reconnu par Alberto del Castillo, on le trouve principalement sur le littoral atlantique. Un seul tesson lui appartient à la grotte d'Achakar, aux environs de Tanger. De Mehdia, viennent les fragments de trois vases dont une jatte (fig. lb). A Dar es Soltane, près de Rabat, un ensemble caractéristique fut recueilli dans un kjoekkenmoedding, sous une couche historique et sur des niveaux paléolithiques: vase à épaulement (fig. 2), nombreux tessons campaniformes, céramique incisée et impressionnée. Dans une industrie lithique et osseuse banale se trouvaient des aiguilles à chas, une spatule dentée en os, utilisable comme peigne pour le décor campaniforme et un brassard d'archer. A proximité, l'abri d'El Menzeh a également fourni des tessons campaniformes. A la grotte des Contrebandiers à Temara, le niveau supérieur renfermait les éléments d'une grande jatte comparable à celle de Mehdia (fig. ld); le campaniforme est également présent à la grotte de l'oued Merzeg. 
Jattes campaniformes
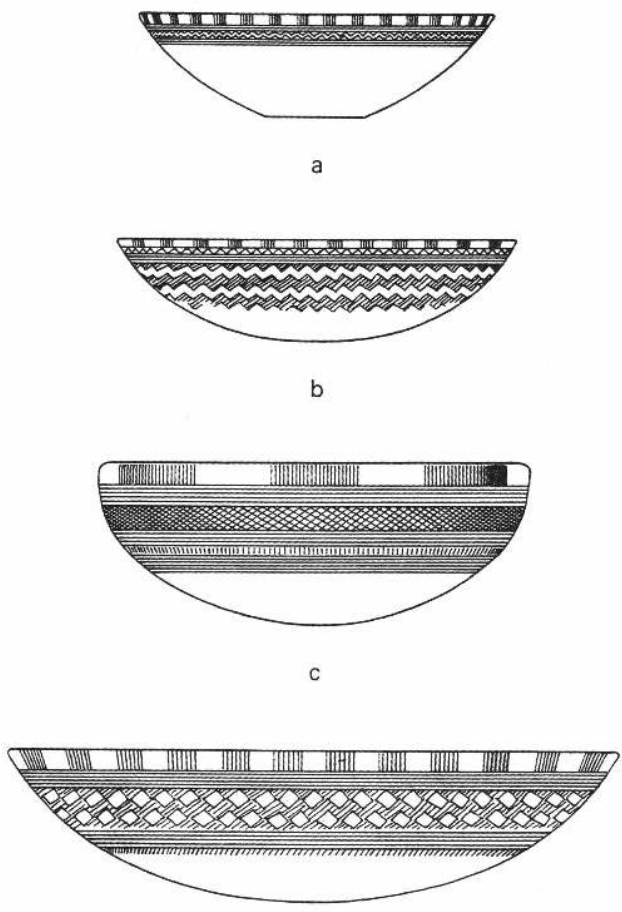

운

$10 \mathrm{~cm}$

d

a : Sidi Slimane ; b : Mehdia ; c ; Palmela (Portugal) ; d : Temara

3 Le rivage méditerranéen du Maroc a livré du Campaniforme dans la grotte de Gar Cahal, près de Ceuta et dans celle de Caf Taht el Gar, à $8 \mathrm{~km}$ de Tétouan, fouillée toutes deux par $\mathrm{M}$. Tarradell. Ce Campaniforme est situé sous un niveau récent et succède à la céramique cardiale ; il est représenté par les éléments de nombreux vases ; les décors y sont variés. 
"Cazuela » de Dar es-Soltane (Maroc) et tessons campaniformes de Rhar oum el Fernan et de l'Oued Saïda (Algérie).
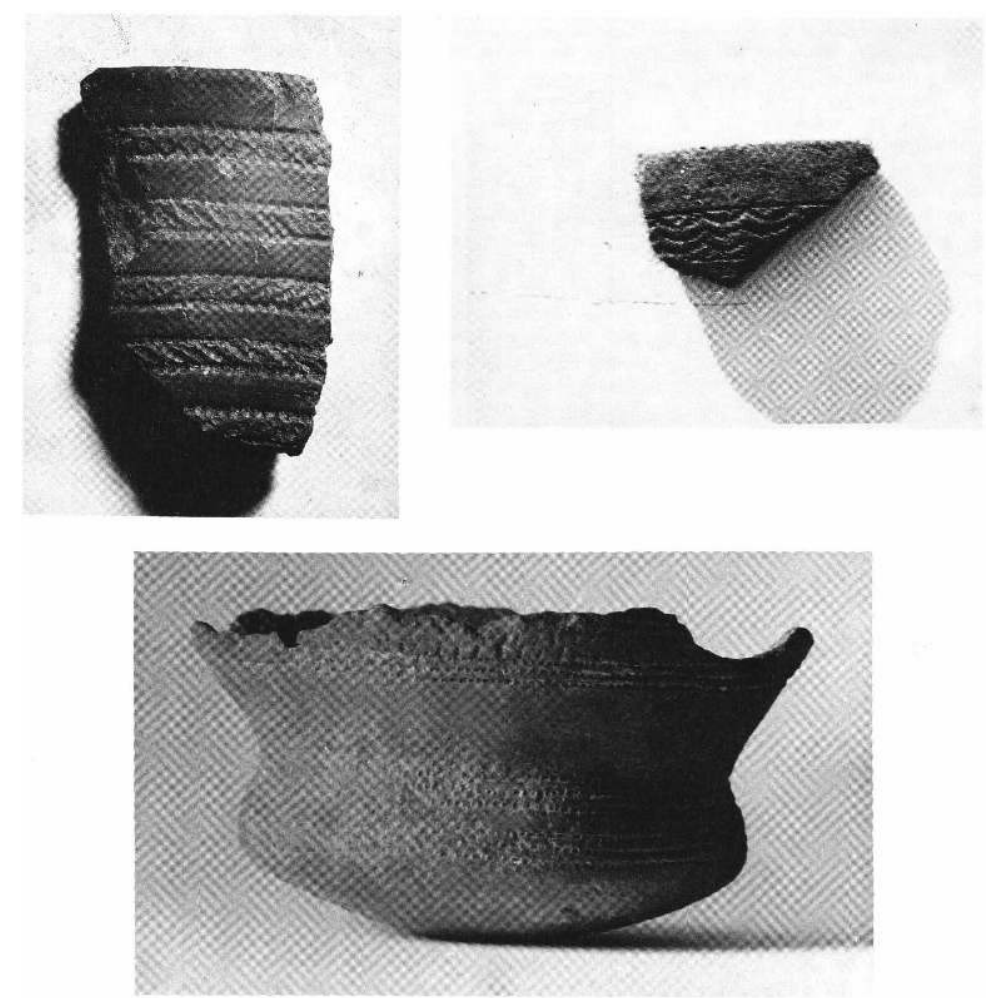

4 L'influence campaniforme n'était pas limitée à quelques points du littoral et à de simples contacts. Elle devait être suffisamment profonde pour introduire des traditions campaniformes dans la céramique locale comme à El Khril, près de Tanger, à la « station de la poterie » de l'oued Mellah ou à la nécropole d'El Kiffen.

5 Mais cette influence s'étendait aussi à l'intérieur des terres. C'est ainsi que deux vases entiers, une cazuela et une jatte (fig. 2a) furent trouvées dans la région de Sidi Slimane, à une soixantaine de kilomètres de la côte. Ils sont soigneusement mais partiellement décorés de lignes horizontales de pointillés enserrant des chevrons. Ces pointillés sont remplis d'une pâte blanche sur la jatte. Enfin la grotte d'Aïn Smene, à $12 \mathrm{~km}$ au sud de Fès a livré trente-six tessons campaniformes dont certains appartenaient à un vase à épaulement.

6 A ces vases ou tessons campaniformes sont souvent associés des outils de tradition chalcolithique en pierre ou en métal ; c'est notamment le cas de plusieurs pointes de Palméla dont l'une est présente à Aïn Smene. Les formes des récipients sont des « cazuelas » et des jattes; il n'y a ni tasse ni vase à pied. Le décor est généralement peu varié ; les motifs sont simples et presque exclusivement obtenus à l'aide d'un peigne denté ; ils n'occupent qu'une partie très limitée de la surface extérieure. 
Répartition des vases campaniformes au Maghreb et dans le sud de la Péninsule ibérique.

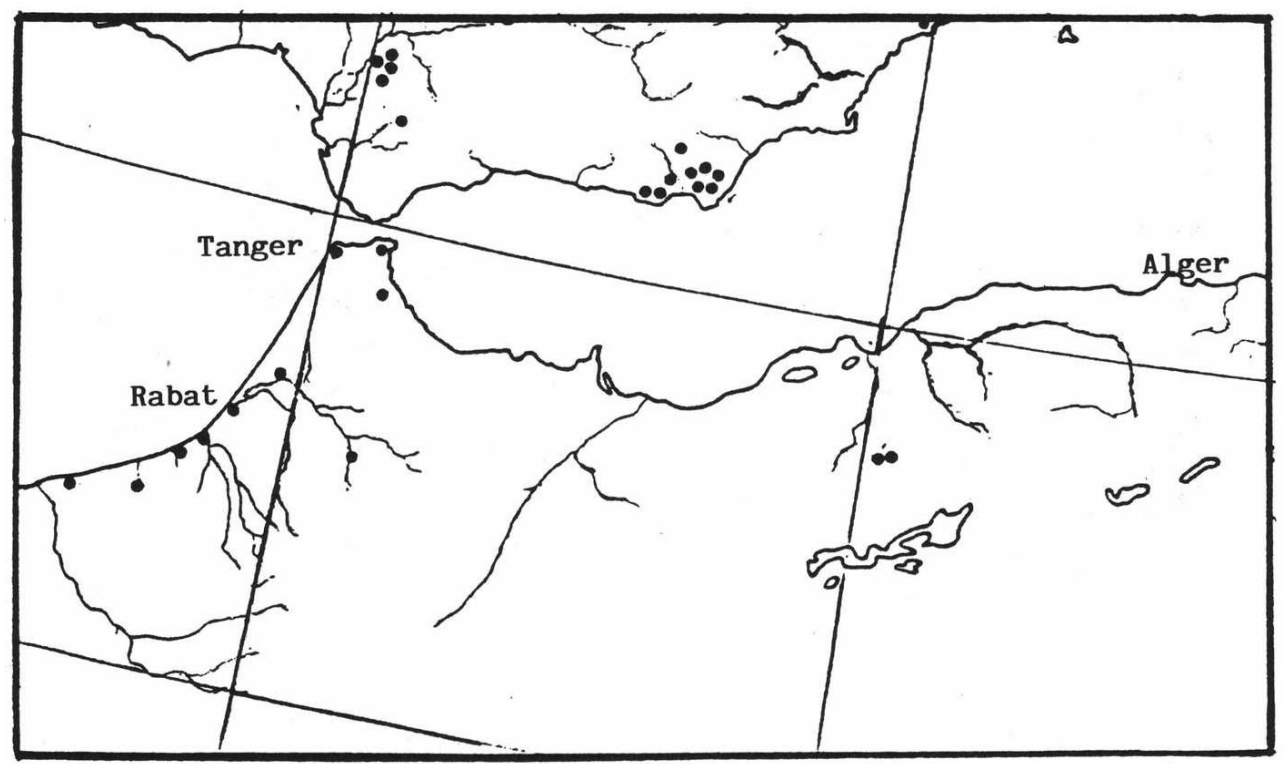

7 Formes et décors s'apparentent à ceux du Portugal (fig. 1 c) et de l'Andalousie. C'est sans aucun doute de la péninsule Ibérique que sont venus les Campaniformes, empruntant la mer d'Alboran et longeant la côte atlantique. Des rivages, ils ont dû pénétrer à l'intérieur du pays par des fleuves comme l'oued Beth ou le Sebou, atteignant ainsi Sidi Slimane et Fès. C'est peut-être par la même voie qu'ils aboutirent en Oranie où furent trouvés les deux seuls tessons campaniformes d'Algérie, à Rhar oum el Fernan et à la grotte de l'oued Saïda, à $120 \mathrm{~km}$ de la côte.

L'infiltration des motifs campaniformes dans la céramique locale, des fabrications sur place suggérées par la présence d'un peigne denté et par la texture de la pâte, laissent supposer qu'une imitation locale a succédé aux importations de la péninsule Ibérique.

\section{BIBLIOGRAPHIE}

CAMPS G., «Les traces d'un âge du bronze en Afrique du Nord », Rev. africaine, t. 104, 1960, p. 32-35.

Id., Aux origines de la Berbérie. Monuments et rites funéraires protohistoriques, Paris, 1961, p. 397-401.

CAMPS-FABRER H., Matière et art mobilier dans la préhistoire nord-africaine et saharienne, Paris, 1966, p. 494-497.

SOUVILlE G., Atlas préhistorique du Maroc, 1, Le Maroc atlantique, Paris, 1973, passim.

Id., « La civilisation du vase campaniforme au Maroc », L'Anthropologie, t. 81, 1977, p. 561-577.

Id., « Témoignages sur l'âge du bronze au Maghreb occidental », C.-R. Acad. Inscriptions et B.-L., 1986, p. 97-114. 
ВОКВОт Ү., Habitats et monuments funéraires du Maroc protohistorique, Aix-en-Provence, 1991, Thèse de doctorat, multigraphié.

INDEX

Mots-clés : Ethnographie 